Itinéraires Itinéraires

Littérature, textes, cultures

2015-3 | 2016

Ethos numériques

\title{
Quand une profession taiseuse se dévoile sur le Web : les ethè discursifs élaborés dans les blogs de policiers
}

When a Silent Professional Group shows Itself on the Web: Discursive SelfPresentation by French Policemen Bloggers

Pierre-Yves Connan, Guillaume Le Saulnier et Benoît Verdier

URL : http://journals.openedition.org/itineraires/3145

DOI : $10.4000 /$ itineraires.3145

ISSN : 2427-920X

Éditeur

Pléiade

Référence électronique

Pierre-Yves Connan, Guillaume Le Saulnier et Benoît Verdier, « Quand une profession taiseuse se dévoile sur le Web : les ethè discursifs élaborés dans les blogs de policiers », Itinéraires [En ligne], 2015-3 | 2016, mis en ligne le 01 juillet 2016, consulté le 01 octobre 2020. URL : http://

journals.openedition.org/itineraires/3145; DOI : https://doi.org/10.4000/itineraires.3145

Ce document a été généré automatiquement le 1 octobre 2020.

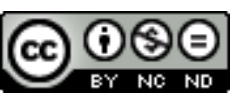

Itinéraires est mis à disposition selon les termes de la licence Creative Commons Attribution - Pas d'Utilisation Commerciale - Pas de Modification 4.0 International. 


\title{
Quand une profession taiseuse se dévoile sur le Web : les ethè discursifs élaborés dans les blogs de policiers
}

\author{
When a Silent Professional Group shows Itself on the Web: Discursive Self- \\ Presentation by French Policemen Bloggers
}

Pierre-Yves Connan, Guillaume Le Saulnier et Benoît Verdier

1 L'exposition de soi sur les interfaces numériques, les expérimentations qu'elle autorise et la réflexivité qu'elle favorise, suscite une abondante littérature. Mais leur appropriation par les groupes professionnels reste, dans une large mesure, un terrain en friche dans la recherche en sciences sociales. En France, la seule étude systématique sur les usages du Web par les collectifs de travail précède l'essor des blogs et des réseaux sociaux (Convert et Demailly 2007). Le dynamisme des enquêtes sociologiques sur l'intégration de l'informatique connectée dans les organisations ${ }^{1}$, ou des études didactiques sur l'appareillage technologique des apprentissages, contraste avec la faible attention consacrée à la «présentation électronique de soi $^{2}$ " (Denouël 2011: 75) comme professionnel et à la mise en récit du monde du travail. Pourtant, l'effervescence observable sur les "médias sociaux» (blogs, forums de discussion, réseaux sociaux, sites de partage) n'épargne pas la sphère productive. De nombreux professionnels s'en saisissent pour narrer leur vie au travail et, ainsi, dévoiler le monde privé des professions. Les plateformes de blogs ${ }^{3}$, en particulier, sont un terrain propice à l'observation de ce phénomène, en vertu de leur diffusion rapide et massive. Certains blogs animés par des travailleurs se sont taillé un franc succès, jusqu'à intéresser les éditeurs. Et ce, jusque dans les professions les plus taiseuses, à l'instar de la police et de la justice ${ }^{4}$.

2 Partant, on se donnera pour objet les écrits d'écran émanant de professionnels, concernant leurs expériences au travail, à destination de lecteurs initiés ou profanes. Plus précisément, on interrogera la reconfiguration des rhétoriques de métiers à la 
faveur de la démocratisation de la parole publique permise par le Web. Au demeurant, tout groupe professionnel procède à une argumentation rationnelle et, conjointement, à une présentation de soi pour «construire la compétence» (Paradeise 1985:18) et revendiquer l'exercice ou le monopole d'une activité. Cette autojustification s'adresse aux pouvoirs publics et à l'environnement sectoriel, mais aussi aux clients et au public. Au-delà des relations en coprésence, elle se loge de plus en plus dans des « interactions médiatisées" (Thompson 2005), c'est-à-dire dans un espace d'interlocution et d'interaction élaboré à distance par un ensemble de médiations sociotechniques.

3 À cette aune, on formulera l'hypothèse que l'appropriation des dispositifs d'autopublication par les professionnels renouvelle les instances, le contenu et les modalités de leurs rhétoriques, en permettant l'affirmation d'une parole publique constituée en marge, voire à l'encontre, des instances traditionnelles de représentation (décideurs, syndicats, porte-parole attitrés). Cela concerne au premier chef les organisations bureaucratisées: les paroles et les apparitions publiques de leurs membres sont ordonnées par un système prégnant de normes et de contraintes, et enchâssées dans un «ordre du discours » (Foucault 1971) qui en réprime les possibilités et en formate les modalités. Cela est d'autant plus sensible avec la généralisation de la communication et de ses impératifs dans les secteurs public et privé. Cependant, la prolifération de discours émis sur le Web par des employés bouscule la division instituée du pouvoir de parler, ainsi que les normes régissant l'expression publique des professions.

4 Pour tester cette hypothèse, on exploitera une étude de cas sur les blogs de policiers, à l'intersection entre sociologie des usages, analyse de discours et sociologie interactionniste des professions. Le corpus a été constitué par une recension à l'aide du moteur de recherche Google, faisant apparaître les supports les plus "populaires », et d'une recherche par "boule de neige », exploitant les référencements croisés entre blogs $5^{5}$. Par souci d'homogénéité, nous avons défini les blogs de policiers de façon restrictive $^{6}$ : on entend par là les blogs édités sans le concours de l'administration par des policiers en exercice, et consacrés pour l'essentiel à leur métier, indépendamment de leur " projet $^{7}$ » d'écriture. Parmi les dix blogs conformes à cette acception sur les dix-neuf recensés ${ }^{8}$, nous en avons retenu cinq par échantillonnage empirique (Bonville 2006 : 101-132). Leur contenu a été " aspiré » pour constituer un corpus stable, avant d'être analysé de façon intensive. Soit un corpus global de 537 billets, publiés entre la date d'ouverture de chaque blog et, par convention, la fin de l'année 2012.

5 Les blogs de policiers sont un matériau privilégié, car ils représentent une transgression à plus d'un titre : ils contournent les restrictions en matière de parole publique et, au-delà, la « culture du secret ${ }^{9}{ }$ (Monjardet $\left.1996: 190\right)$ en vigueur dans l'institution ; ils contrarient ses efforts pour contrôler l'expression des policiers sur les médias sociaux, et pour inscrire ceux-ci dans une politique de communication globale ${ }^{10}$ ; au surplus, ils émanent de «simple[s] policier[s] de base " (selon la dénomination utilisée par un policier blogueur), alors que le genre autobiographique concerne en priorité les commissaires ou les flics légendaires (Ocqueteau 2006 : 53-82). En effet, les cinq blogueurs analysés disent appartenir au corps des gardiens de la paix, subalterne dans la hiérarchie des corps, et s'expriment sous pseudonyme. Soit un questionnement à la fois sur le dit et le dire : comment les blogs de policiers donnent-ils à voir la police, son rôle, son travail ? Comment leurs auteurs s'y prennent-ils pour revendiquer un titre à parler et, ainsi, justifier leur prise de parole? 
6 Pour y répondre, on examinera les ethè $\grave{11}^{11}$ discursifs construits dans et par les écrits d'écran, comme autant de stratégies énonciatives pour établir un lien avec les lecteurs, mais aussi pour se hisser au rang d'énonciateur légitime et prétendre parler de et au nom de la profession. À cette fin, on exploitera une analyse de contenu manuelle, pour identifier les aspects du métier mis en avant ou au contraire relégués au second plan, ainsi qu'une analyse de discours à l'aide du logiciel Alceste, pour objectiver les " mondes lexicaux ${ }^{12}$ » formés par la distribution du vocabulaire et leurs relations. En complément, on s'appuiera sur des entretiens approfondis auprès de trois policiers blogueurs, pour interpréter le matériau textuel à la lumière de leurs trajectoires biographiques et professionnelles. On précisera le contenu et le projet des blogs étudiés, qui sont tous des témoignages, avant de détailler les différents ethè élaborés pour soutenir cette visée testimoniale.

\section{Des témoignages exemplaires à partir d'ethè complémentaires}

7 Le corpus constitue, à bien des égards, un matériau hétérogène : Que fait la police? (52 billets entre 2006 et 2012) propose des récits d'interventions en sécurité publique ; Flic2rue (53 billets de 2008 à 2012) ainsi que Vie de flic! (103 publications depuis 2008) y ajoutent l'autobiographie; PJ en capitale (103 billets depuis 2009) conjugue récit de vie en police judiciaire et montées en généralité sur l'actualité policière et la politique pénale ; Police-histoires (226 billets de 2008 à 2012) narre « les histoires du quotidien des flics » à travers la fiction ${ }^{13}$. Mais, par-delà leurs spécificités, ces blogs relèvent tous du témoignage, entendu comme un régime discursif doté de ses modalités propres. Cette visée testimoniale est explicite dans le titrage des supports, la catégorisation des billets ("humeur», "brèves de flics", "anecdotes, histoires vécues») ou encore la présentation des auteurs («Ce blog est le modeste témoignage de ma vie de flic [...]. Bref, voici la vie d'un homme ordinaire qui exerce un métier extraordinaire... $\left.{ }^{14} »\right)$. Les récits se veulent référentiels: ils renvoient à des événements supposément avérés, énoncés par un locuteur à la fois témoin et garant de leur existence, et attestés par une "certification autobiographique" (Dulong 1998). Seule exception, le blog PH revendique néanmoins, sous les tours narratifs et les effets de style, une fiction résolument réaliste, manifestée par un récit « authentifiant » (Jost 1997).

8 Ces récits expérientiels à la première personne exploitent un vécu, ou plutôt un «revécu» (Politanski 2009: 255), pour narrer le quotidien des auteurs et, par extension, de leurs pairs. Si les expériences sont éprouvées et rapportées par un locuteur, leur assemblage compose à chaque fois un portrait (individuel et de groupe) donné comme révélateur de ce que vivent les flics. D'ailleurs, plus d'un quart des billets (27,5\%) évoquent ouvertement la profession, ou du moins tel ou tel de ses segments. Le témoignage personnel a valeur d'exemple: il sert de support et de prétexte pour produire un discours sur la police, et sensibiliser autrui aux réalités et aux difficultés de la profession. Sous cet angle, les billets répondent à une rhétorique de l'« exemplum »: ils sont autant de brefs récits présentés comme véridiques et mobilisés à des fins d'édification, en vertu du présupposé selon lequel «ce qui est valable pour l'un est valable pour tous ceux qui partagent sa situation ${ }^{15} »$. Cette visée d'édification est souvent explicitée par les auteurs : 
Voici l'historique de mes chroniques de la plus récente à la plus ancienne. Toutes ces anecdotes sont (malheureusement) authentiques et sont l'expression d'interventions banales pour le simple «policier de base » que je suis. Je ne doute pas que beaucoup de "frères d'armes", de passage ici, se reconnaittront dans ces «moments de police»; si je n'ai rien à prouver à ces derniers, j'espère (naïvement peut-être) donner une autre idée de ce métier difficile aux autres lecteurs, d'où qu'ils viennent, quels qu'ils soient... (Présentation du blog QFP)

Le témoignage revêt ici des formes variables, moins dans le contenu que dans les modalités du discours et, notamment, les modes de figuration de l'auteur. L'analyse à l'aide du logiciel Alceste permet d'inférer différents ethè, à partir des classes d'énoncés définies statistiquement comme les plus spécifiques du corpus. Le vocabulaire se distribue parmi sept classes, émanant de deux sous-ensembles. D'un côté, quatre classes correspondent au témoignage in situ, de l'intérieur de la police sinon dans le feu de l'action. Elles contribuent à la construction de l'ethos du témoin. Celui-ci se double parfois de l'image du confident, dans un effeuillage du moi diversement dosé. De l'autre, trois classes font apparaître des «postures énonciatives" (Reinert 2007) plus distanciées: l'ethos du spécialiste, dont le savoir professionnel justifie un discours évaluatif sur la police, et celui de l'auteur, dans la mise en scène du travail d'écriture. Chaque blog mobilise et articule, à des degrés divers, plusieurs ethè à la fois.

Les classes d'énoncés du corpus identifiées par le logiciel Alceste (échantillon)

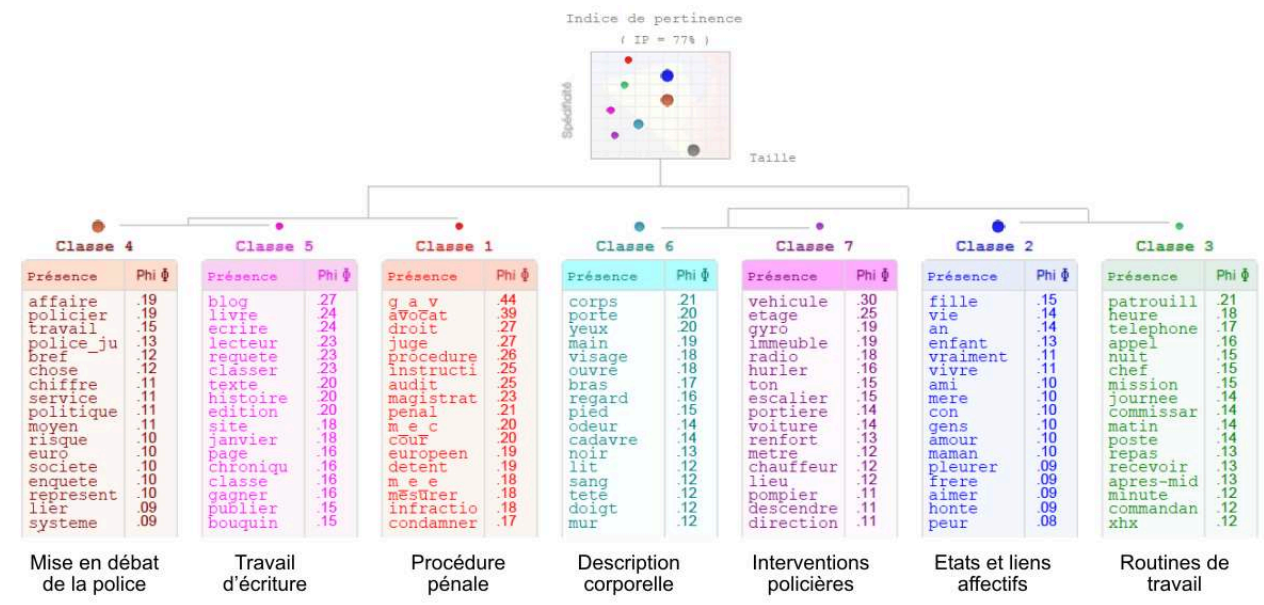

Clé de lecture : les énoncés significatifs de chaque classe sont regroupés, par classification descendante hiérarchique, en vertu de leur co-occurrence. Ils sont dotés d'un coefficient Phi, qui mesure la force du lien entre le mot et la classe ; un coefficient Phi supérieur à .20 indique un lien fort. L'analyse automatique distingue les deux sous-ensembles les plus contrastés, eux-mêmes divisés en classes plus réduites.

\section{L'ethos du témoin}

10 Le premier ethos qui réalise et autorise la mise en récit de la profession est celui du témoin. Le locuteur prend la parole sous un double statut : comme témoin oculaire des événements rapportés, mais aussi comme premier intervenant. Le récit est égocentré, fortement modalisé, et donne à voir la police en vue subjective : le lecteur est projeté dans la peau de l'auteur. La narration se concentre en priorité sur les activités assumées par l'auteur ( $60 \%$ des billets) et les interventions données comme ordinaires. Elle se structure en général selon un schéma actantiel ternaire, où se joue un face-à- 
face entre l'auteur, entouré de ses collègues de travail, et les «clients ${ }^{16}$ » (respectivement 82,6\%, 50,4 \% et 53,2 \% des billets).

11 Cet ethos se construit sur trois thèmes récurrents. D’abord, les routines de travail (classe 3) sont décrites avec insistance: les lieux («commissariat», "poste», « accueil», «ville»), les interlocuteurs ( chef», « commandant», «brigade», « commissaire ») et les activités ordinaires («patrouille», « mission», « appel», « conversation ", « rendez-vous ", " repas », " café ») sont mobilisés de concert pour planter le décor. De même, les indicateurs de temps sont surreprésentés («heure», « nuit », « journée », « matin », « après-midi », " vacances »), parfois avec une précision d'horloger. Ensuite, les blogs étudiés font la part belle aux récits d'interventions (classe 7). L'action prend place dans un décor urbain ("étage ", " immeuble », " escalier», " parking»), et montre la police comme force de projection motorisée ("véhicule », " gyrophare », « deux tons », " portière », « voiture », « renfort ", «chauffeur»). Les marqueurs d'une relation spatiale ou temporelle, mais aussi les verbes d'action («hurler», « descendre», « rouler», « approcher», « claquer», « concentrer ») abondent à l'intérieur de phrases courtes, rendant palpables la tension nerveuse et les montées $d$ '«adrénaline». Enfin, les scènes macabres (classe 6) représentent une figure imposée. La découverte (" porte », " ouvrir », « noir », " lit », "soudain ») et la manipulation de cadavres (" regarder", " refermer», " poser », «doucement ») donnent matière à une surenchère de détails morbides («corps», « yeux ", « main », « visage ", « bras ", « pied », « odeur ", « cadavre ", « sang ", " tête ", «bruit », " crâne », " pantalon », «torse », " rouge »), afin de produire un effet dramatique ou, à l'occasion, comique. Ce ressort narratif culmine avec les récits d'autopsies, volontiers nauséeux.

Pareils référents sont propices à un dévoilement des états du moi, dans un discours où la subjectivité gouverne ( Je ne vais pas rentrer dans le détail des faits [...]. Mais je peux vous parler de ressenti, ce qu'il se passe dans la tête de l'enquêteur $\left.{ }^{17} »\right)$. Les modalisateurs sont légion et les émotions affleurent. Jusqu'à $42 \%$ des billets témoignent des affects ressentis par les auteurs : l'excitation d'endosser l'uniforme et l'émulation collective voisinent avec les moments de fatigue et de découragement, mais aussi avec l'effroi ou la peine face aux interventions éprouvantes. Le récit de la police au quotidien se donne ainsi comme une description à fleur de peau des « éboueurs de la société » (selon une expression indigène), de leurs états et leurs traumas. Il réside également dans la compassion exprimée à l'égard des policiers morts en service. Cette prégnance des affects fait basculer le récit, par endroits, du témoignage à la confidence.

\section{L'ethos du confident}

13 Les policiers blogueurs sont témoins de situations traumatisantes, qu'ils estiment méconnues du public et qui ne les laissent pas indemnes. À cette aune, leurs écrits d'écran ont une double fonction. Ils servent de médiation pour divulguer un «savoir coupable » (Hughes 1996 : 99-106) sur les déviances et les souffrances du corps social, pour dire l'indicible et le partager au-delà des seuls initiés. Parallèlement, ils permettent d'extérioriser des expériences douloureuses, professionnelles mais aussi personnelles, pour mieux les apprivoiser. De façon significative, les auteurs se sont investis durablement dans l'écriture d'un blog après une expérience biographique marquante, survenue au travail (F2R, PJEC) ou en dehors (PH, VDF), et révélée tôt ou tard 
aux lecteurs. Les blogs de policiers fournissent dès lors le prétexte à un dévoilement de l'intime, dans la verbalisation des souffrances au travail ou des épreuves biographiques. Parmi eux, l'auteur de F2R confirme en entretien la fonction cathartique de son activité d'écriture :

Moi, toutes les histoires racontées sont des histoires vécues. [...] C'est l'action policière qui me donne envie d'écrire. Par exemple le dernier billet, «Sophie ", où je raconte une intervention qui m'a marqué. C'est l'intervention qui décide si j'écris ou pas, selon ce que j'ai ressenti. C'est souvent sur des interventions qui m'ont dérangé, j'écris pour mettre de la distance. (Entretien avec l'auteur de F2R)

Cet ethos $d u$ confident s'exprime avant tout dans l'évocation des liens et des déchirements familiaux (classe 2). La famille apparaît comme un refuge affectif mais aussi, par contraste, comme le théâtre de drames insoupçonnés. L'immersion dans l'intimité des familles («fille ", « enfant », «ami », «mère », «maman », «frère ») confronte les policiers à des émotions aussi vives que contradictoires («con», " amour ", " pleurer ", « aimer ", « honte », «peur »), parfois attisées par un détail anodin («belle», "photo»). Les récits d'interventions sur des cas de violences intrafamiliales, dont des enfants sont témoins ou victimes, sont un thème privilégié pour énoncer et exorciser les affects enkystés.

Le blog VDF est le plus symptomatique de cette pente intimiste. L'auteur évoque longuement sa vie privée ( $28 \%$ des billets). À tel point qu'elle se confond avec sa «vie de flic " dans un tiers des billets : sa vocation policière découle "naturellement » de son enfance traumatisée, tandis que son existence personnelle est rythmée par les hauts et les bas de sa carrière. Ces confidences biographiques, inscrites dans un blog servant d'exutoire ("écrire me permet de soulager ma conscience ${ }^{18}$ "), se logent également dans les nombreux échanges avec les lecteurs (trois commentaires par billet en moyenne) : ils font la part belle aux messages de réconfort ou d'encouragement, dans un discours volontiers psychologisant où le tutoiement s'impose («Quelle belle revanche sur la vie! Tu peux vraiment être fier de toi...19 ${ }^{19}$ ). À l'opposé de cette mise à $\mathrm{nu}$, les policiers blogueurs façonnent leur ethos par un discours plus distancié affirmant leurs compétences.

\section{L'ethos du spécialiste}

16 Raconter le monde policier de l'intérieur suppose un titre à parler, c'est-à-dire d'apparaître comme un locuteur légitime au discours crédible. En l'occurrence, les policiers blogueurs se présentent tous comme des flics " de terrain ", en prise directe avec la bêtise et la violence ordinaires, la délinquance de masse et la misère sociale. À ce titre, ils font voir et valoir un savoir pratique sur les délinquants, occasionnels ou professionnels, par une description minutieuse de leurs habitudes, leur jargon, leurs modes opératoires. C'est notamment le cas dans les récits de maraudes, où les auteurs de F2R et $Q F P$ filent la métaphore du «chasseur » guidé par son instinct et toujours à l'affût :

Sans être nyctalope, j'ai appris à regarder dans le noir, à discerner dans l'obscurité, à comprendre les ombres. J'aime chasser au clair de lune, repousser mon sommeil pour veiller sur celui des autres, errer librement au gré de mes instincts tel le hibou qui voit tout. ( Oiseau nocturne », F2R, 11 février 2008)

17 Cet ethos du spécialiste est particulièrement accusé dans le blog PJEC. L'auteur se présente comme un professionnel du travail d'enquête, "qui [pratique] le droit à 
longueur d'année ». Il affiche ostensiblement, dans la présentation du blog, son appartenance au prestigieux 36 quai des Orfèvres. Cette spécialisation apparaît surtout dans les billets, où "Chris PJ" s'empare d'affaires traitées personnellement ou prélevées dans l'actualité pour produire une pédagogie du droit (classe 1). Il explique en détail la procédure pénale (" gard-é-e à vue », « droit », « instruction », "audition », " mis-e en cause », « détention ", « mis-e en examen »), en se référant aux textes de loi ("code de procédure pénale», "convention européenne» des droits de l'homme, " décision », " disposition ») et aux autorités judiciaires (" juge », « Cour européenne », "parquet", "Conseil constitutionnel»). Ces montées en généralité, propices à un " effacement énonciatif» (Amossy 2010: notamment 183-208), cohabitent avec un discours évaluatif sur la politique pénale et les réformes en cours. L'auteur désigne ainsi la réforme de la garde à vue, à laquelle il s'oppose à quinze reprises, comme "[son] cheval de bataille ${ }^{20}$ ». Son expertise autorise un dialogue indirect avec les professionnels de la justice ("avocat», «magistrat »), constitués comme une instance énonciative incarnant des positions concurrentes.

Ce savoir professionnel (théorique ou pratique) fonde la prétention à instruire les profanes, mais aussi à combattre les stéréotypes réducteurs (mentionnés dans 16,7\% des billets). Les auteurs ont à cœur de réhabiliter la profession, tant dans sa dimension pastorale de "dernier rempart» face au délitement du lien social, que dans ses fonctions répressives, «afin de sensibiliser les contrevenants à l'utilité et au quotidien de l'agent verbalisateur ${ }^{21}$ ». Ce travail de contre-stéréotypage est poussé à l'extrême dans le blog QFP, où il occupe $50 \%$ des billets. L'auteur manie avec ironie les clichés et interpelle directement les lecteurs, pour fustiger les préjugés "anti-police» et l'angélisme en vigueur parmi la population:

La petite fleuriste a appelé à l'aide et la police est venue. Une fleuriste, seule dans son magasin, qui s'est fait rafler son fond de caisse par une bande de roms. Ah. J'ai vu l'étincelle dans tes yeux. Lecteur. [...] Les policiers sont arrivés, avec leur gyrophare, leurs flingues, leur envie de sauver la veuve, l'orphelin et la fleuriste ; mais que nenni, ils contrôlaient gratuitement des nécessiteux, là, en plein milieu de la place commerçante bondée de passants ignares et indignés, aux yeux exorbités. [...] Alors des policiers racistes, arrivés comme des cowboys, étaient venus contrôler des pauvres gens parce qu'ils étaient roms. («La petite fleuriste », QFP, 6 novembre 2012)

19 L'ethos du spécialiste accompagne l'affirmation d'une éthique du travail. Les auteurs donnent l'image de policiers investis dans leur travail, agissant au nom de la protection des personnes, à commencer par les plus vulnérables, de la réparation judiciaire due aux victimes, et de la recherche de la vérité. Ils sont épaulés par leurs pairs, omniprésents dans le récit: l'analyse automatique dénombre jusqu'à 561 occurrences pour le mot collègue. Les situations narrées donnent matière à définir subjectivement, et à mettre en valeur, les rôles de "flic de rue ", d'enquêteur, de chef de groupe. À un degré supérieur, les blogs $F 2 R, P J E C$ et VDF produisent un récit vocationnel, assimilable à une profession de foi ( Aider les gens, évoluer, ne pas vivre dans la routine, connaître davantage la vie, la mort, le danger, l'adrénaline... La police nationale vint à mon esprit aussi naturellement que le jour se lève $\left.{ }^{22} . »\right)$

Mais cette éthique du travail se heurte aux moyens disponibles et aux consignes prescrites (évoqués dans un dixième des billets), tandis que les vocations peuvent être contrariées sinon broyées par une bureaucratie «immuable ${ }^{23}$ ». Le corpus installe d'un bout à l'autre une opposition entre, d'un côté, un discours de la professionnalité, dans 
l'affirmation des normes et des valeurs censées régir les policiers, et, de l'autre, un discours de l'instrumentalité, dans le rappel des contraintes et des "servitudes" qui freinent leur action. On trouve un résumé de cette contradiction sous la plume de l'auteur de $F 2 R$ : «Si je pense être un bon policier, je suis certain d'être un piètre fonctionnaire ${ }^{24}$. » De fait, l'ethos du spécialiste sert d'opérateur privilégié pour formuler des doléances: il procure des ressources argumentatives pour contester, en connaissance de cause, le fonctionnement de l'appareil policier ainsi que les orientations de la politique pénale. L'auteur de PJEC relaye le débat public sur la police et la justice (classe 4), et prend position en vertu de ses compétences: il commente l'actualité policière et judiciaire ("affaire", "policier», "police judiciaire", " enquête », "journaliste»), mais aussi la politique pénale et les réformes engagées (« chiffre», «politique», «moyen», « risque», « société », « administration», " pouvoir »). De même, les auteurs de F2R et QFP arguent de leur expérience du terrain, le premier pour déplorer les « injustices » de l'administration, le second pour dénoncer sans relâche l'absence de réponse pénale, l'impunité des délinquants et l'impuissance des policiers.

\section{L'ethos de l'auteur}

21 Les policiers blogueurs exhibent un savoir professionnel, mais aussi une compétence d'écrivain. Leur témoignage se donne comme véridique, mais il ne sacrifie pas pour autant toute prétention littéraire. À mille lieues des conventions rigides de l'écriture policière, les effets stylistiques sont utilisés et parfois revendiqués pour restituer au mieux les événements, leur intensité, et gagner tant l'attention que l'empathie des lecteurs ${ }^{25}$. Pareils desseins justifient la confusion des genres, savamment dosée, entre le vrai et le vraisemblable. L'ethos construit par les policiers blogueurs recouvre ainsi, parmi ses divers replis, le statut d'écrivain amateur, suffisamment habile pour narrer le monde policier dans ses aspects les plus typiques comme les plus banals, camper des personnages et des situations crédibles, donner vie à des artefacts textuels et, finalement, fidéliser une communauté de lecteurs.

Cet ethos d'auteur se manifeste dans une recherche stylistique assumée. Le blog F2R, devenu un ouvrage imprimé à l'aide d'une société d'autoédition, comporte notamment plusieurs billets rédigés en slam, sublimant des thèmes courants par le rythme et les rimes. De même, les récits d'interventions rapportés dans QFP montrent le quotidien des policiers, rehaussé par une écriture soignée. Les prétentions littéraires concernent au premier chef $P H$, édité sous le pseudonyme «Serge Reynaud». Le projet du blog, explicité dans le premier billet, consiste à publier des «bonnes histoires ${ }^{26}$ » sur la police, rédigées par l'auteur ou, en de rares cas, sélectionnées par ses soins. Il revendique une licence littéraire ( Je ne revendique pas le réel pour absolu : j’écris ${ }^{27}$ ») et, simultanément, un récit authentifiant directement inspiré d'expériences vécues. Surtout, il s'affranchit de façon régulière du récit policier pour narrer son travail d'écrivain. Ces incursions dans la création littéraire (classe 5) se matérialisent par un vocabulaire spécifique : «blog ", « livre ", « écrire ", « lecteur », « texte », « histoire ",

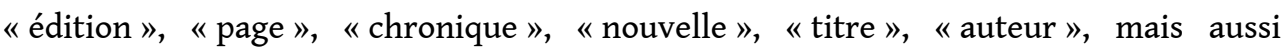
" gagner », « concours », « publier », « bouquin », « vendre », « exemplaire ». L'auteur se présente comme un autodidacte, épris de la langue française et intransigeant envers les fautes d'orthographe. Il affirme progressivement sa vocation d'écrivain, encouragée 
et confirmée par les commentaires laudatifs des lecteurs, les rencontres et les récompenses glanées dans des concours de nouvelles, jusqu'à la publication de deux recueils de textes extraits de son blog et l'invitation à des séances de dédicaces. Cette image de nouvelliste apparaît dans la rédaction des billets, par leur style et leurs qualités littéraires. Mais elle réside, tout autant, dans un métadiscours récurrent sur le procès et les tracas de la création littéraire, où l'auteur démontre, exemples à l'appui, sa maîtrise des rouages narratifs et des effets de réel. Elle est encore réaffirmée dans les messages échangés avec Bénédicte Desforges, auteure à succès d'un blog et de deux ouvrages testimoniaux consacrés à son expérience de policière, dans un procédé de légitimation croisée au bénéfice de l'un et de l'autre.

\section{Conclusion}

Les blogs de policiers étudiés se veulent des témoignages authentiques sur le monde policier. Pour satisfaire cette visée testimoniale, leurs auteurs s'appuient sur un ethos composite, qui conjugue plusieurs postures énonciatives. Chacune fournit des ressources discursives pour façonner l'image des locuteurs et orienter la réception des lecteurs, mais aussi pour justifier une prise de parole donnant à voir la police et les policiers de l'intérieur, en l'absence de tout titre à parler officiel. Le statut de témoin se construit dans ces stratégies énonciatives et, conjointement, dans l'espace d'interlocution établi par les échanges entre auteurs et lecteurs ${ }^{28}$ : en effet, des pairs déclarés interviennent dans les commentaires pour décerner un certificat d'authenticité au témoignage des policiers blogueurs. Les dispositifs d'auto-publication fonctionnent ainsi comme un support de réflexivité individuelle et collective. Dans le même temps, ils offrent un espace interstitiel de réappropriation du pouvoir de parler, dans une profession rigoureusement tenue au silence, dont la parole est monopolisée, sinon confisquée, par les porte-parole attitrés. Cela se manifeste, notamment, dans la quasi-absence des syndicats policiers au sein du corpus (sept occurrences seulement), tant dans la présentation des auteurs que dans leur témoignage, alors que deux d'entre eux exercent (PJEC) ou ont exercé (F2R) des fonctions syndicales, et que les conditions de travail et la politique pénale sont des thèmes récurrents.

Par-delà les spécificités de son objet, cette étude de cas plaide en faveur d'une définition extensive des rhétoriques professionnelles et de leur écologie, intégrant les écrits d'écran produits et commentés par les professionnels, en deçà des discours institutionnels et de leur "lissage " (Oger et Ollivier-Yaniv 2006), ou des discours syndicaux et de leurs figures imposées. Par leur foisonnement, leur accessibilité et leur inventivité, ces productions discursives sont un matériau privilégié pour observer la mise en récit et les formes de subjectivation des rapports de production. Pareille appropriation, par les professionnels, des moyens d'affirmer et, potentiellement, de resignifier ce qu'ils sont et font institue le Web comme une instance sui generis d'élaboration et de validation des identités au travail, mais aussi de politisation et de mobilisation corporatives ${ }^{29}$. 


\section{BIBLIOGRAPHIE}

Amossy, Ruth, 2010, La Présentation de soi. Éthos et identité verbale, Paris, PUF, coll. « L'interrogation philosophique ».

Bonville, Jean de, 2006, L'Analyse de contenu des médias. De la problématique au traitement statistique, Bruxelles, De Boeck \& Larcier, coll. « Culture \& Communication ».

Canu, Roland et Datchary, Caroline, 2010, « Journalistes et lecteurs-contributeurs sur Mediapart. Des rôles négociés ", Réseaux, nº 160-161, p. 195-223.

Cardon, Dominique et Delaunay-Téterel, Hélène, 2006, « La production de soi comme technique relationnelle. Un essai de typologie des blogs par leurs publics ", Réseaux, nº 138, p. 15-71.

Connan, Pierre-Yves, Le Saulnier, Guillaume et Verdier, Benoît, 2013, « Les rhétoriques professionnelles au défi des blogs. Fragmentation du discours et stratégies de légitimation », dans B. Vacher, C. Le Moënne et A. Kiyindou (dir.), Communication et débat public : les réseaux numériques au service de la démocratie ?, Paris, L'Harmattan, coll. « Communication et civilisation », p. 147-154.

Convert, Bernard et Demailly, Lise, 2007, Les Groupes professionnels et l'Internet, Paris, L'Harmattan, coll. « Logiques sociales ».

Denouël, Julie, 2011, « Identité », Communications, nº 88, p. 75-82.

Dulong, Renaud, 1998, Le Témoin oculaire. Les conditions sociales de l'attestation personnelle, Paris, Éditions de l'EHESS, coll. « Recherches d'histoire et de sciences sociales ».

Foucault, Michel, 1971, L'Ordre du discours, Paris, Gallimard.

Hughes, Everett, 1996, Le Regard sociologique. Essais choisis, Paris, Éditions de l'EHESS, coll. « Recherches d'histoire et de sciences sociales».

Jost, François, 1997, « La promesse des genres », Réseaux, n 81, p. 11-31.

Jost, François, 2004, « Séries policières et stratégies de programmation », dans P. Beylot et G. Sellier (dir.), Les Séries policières, Paris, L'Harmattan, INA, coll. « Les médias en actes », p. 57-83.

Kessous, Emmanuel et Metzger, Jean-Luc, 2005, Le travail avec les technologies de l'information, Paris, Lavoisier, Hermès Science Publications, coll. « Technique et scientifique des télécommunications ».

Klein, Annabelle (dir.), 2007, Objectif blogs ! Explorations dynamiques de la blogosphère, Paris, L'Harmattan, coll. « Communication et civilisation ».

Monjardet, Dominique, 1996, Ce que fait la police. Sociologie de la force publique, Paris, La Découverte, coll. « Textes à l'appui ».

Ocqueteau, Frédéric, 2006, Mais qui donc dirige la police ? Sociologie des commissaires, Paris, Armand Colin, coll. « Sociétales ».

Oger, Claire et Ollivier-Yaniv, Caroline, 2006, « Conjurer le désordre discursif. Les procédés de "lissage" dans la fabrication du discours institutionnel ", Mots. Les langages du politique, $\mathrm{n}^{\circ} 81$, p. 63-77.

Paradeise, Catherine, 1985, « Rhétorique professionnelle et expertise », Sociologie du travail, vol. $27, \mathrm{n}^{\circ} 1, \mathrm{p} .17-31$. 
Politanski, Pascal, 2009, «L'identité professionnelle des enseignants exprimée à travers l'internet », dans J.-Y. Causer, J.-P. Durand et W. Gasparini (dir.), Les Identités au travail. Analyses et controverses, Toulouse, Octarès, coll. « Le travail en débats », p. 251-258.

Reinert, Max, 2007, « Postures énonciatives et mondes lexicaux stabilisés en analyse statistique de discours ", Langage et société, $\mathrm{n}^{\circ}$ 121-122, p. 189-202.

Soubrié, Thierry, 2008, « Images de soi dans un blog professionnel d'enseignants stagiaires », Alsic, vol. 11, n 1, p. 121-149, [En ligne], http://alsic.revues.org/843.

Thompson, John B., 2005, « La nouvelle visibilité », Réseaux, nº 129-130, p. 59-87.

\section{NOTES}

1. Voir notamment Kessous et Metzger (2005).

2. On mentionnera, toutefois, les travaux sur les images de soi projetées dans les blogs d'enseignants ou de policiers (Soubrié 2008, Politanski 2009, Connan, Le Saulnier et Verdier 2013), ainsi que ceux sur la redéfinition des compétences et des identités des journalistes professionnels liée à la diffusion des pratiques d'auto-publication (Canu et Datchary 2010).

3. Un blog est un dispositif d'auto-publication caractérisé par une facilité d'appropriation, des écrits multimodaux présentés dans un ordre ante-chronologique, une lecture hypertextuelle et une interactivité auteurs-lecteurs.

4. Voir notamment : les récits testimoniaux de l'« ex-flic» Bénédicte Desforges, et des avocats Maître Eolas et Maître Mô; les blogs d'expertise critique de Georges Moréas et Philippe Bilger, respectivement commissaire et magistrat honoraires.

5. La combinaison de ces deux techniques a permis de neutraliser leurs biais respectifs : à commencer par les logiques internes de tout dispositif d'indexation, pour la première, et par les formes horizontales de cooptation entre blogueurs, pour la seconde.

6. Cela exclut les blogs hébergés sur le site du ministère de l'Intérieur à des fins de communication recrutement, ainsi que les blogs personnels rédigés par d'anciens policiers. Ces derniers sont, par leurs spécificités (statut et stature de l'auteur, liberté de parole, volume de la production), un objet d'étude à part entière.

7. Cette notion descriptive (Klein 2007 : 37-68) synthétise deux dimensions essentielles des blogs : le degré d'implication des auteurs dans les contenus publiés, et le réglage de l'interactivité avec les lecteurs.

8. Les blogs recensés vont du témoignage sur le métier à la fiction réaliste, en passant par la veille des actualités policières, le commentaire sérieux ou satirique de l'information générale, ou encore le récit de vie en congé paternité d'un " poulet qui devient papa-poule ».

9. Les policiers sont astreints au devoir de réserve, à l'obligation de dignité, à la discrétion et au secret professionnels et, plus spécifiquement, au secret de l'enquête et de l'instruction. L'accès à la parole publique est monopolisé par les autorités et les syndicats policiers.

10. Entretien avec les chefs des services communication à la Direction générale de la Police nationale (DGPN) et à la Préfecture de police de Paris (PP). Ainsi, le «Code de déontologie » de la police et de la gendarmerie de janvier 2014 étend l'obligation de dignité aux publications sur les réseaux sociaux (article R. 343-12).

11. Nous reprenons ici le concept d'ethos développé par Ruth Amossy (2010), à l'articulation entre construction identitaire et fonction argumentative.

12. Sur les assises théoriques et les vertus heuristiques du logiciel Alceste, voir notamment Reinert (2007).

13. Désormais, les blogs étudiés seront respectivement désignés ainsi : QFP, F2R, VDF, PJEC, PH. 
14. Présentation du blog VDF.

15. François Jost (2004 : 74) montre l'actualité de l'exemplum, figure rhétorique héritée du Moyen Âge.

16. La catégorie de « clients » regroupe les requérants, les victimes et les auteurs d'infractions.

17. « Le destin est ainsi fait... », PJEC, 21 janvier 2012.

18. « Mon nouveau nid! », VDF, 20 septembre 2009.

19. "Naissance d'une vocation", VDF, 26 décembre 2008: commentaire d'un lecteur daté du 22 août 2009.

20. Entretien avec l'auteur de PJEC.

21. "Street marketing préventif à caractère d'intérêt national », F2R, 31 mars 2010.

22. « Naissance d'une vocation », VDF, 26 décembre 2008.

23. «Immuable », F2R, 31 juillet 2008.

24. « Sanction par équité », F2R, 13 janvier 2009.

25. Entretien avec l'auteur de $P H$.

26. «On se lance!", PH, 16 mai 2008.

27. « Faut que ça sorte! ", PH, 6 août 2008.

28. Nous ne pouvons, ici, développer davantage les processus d'interlocution, aussi essentiels soient-ils dans le dispositif des blogs et la construction des ethè.

29. On notera, par exemple, l'émergence de "proto syndicats" en ligne dans la police (le "Collectif libre et indépendant de la police», vite sabordé) et la gendarmerie (le forum de discussion «Gendarmes et Citoyens", largement plébiscité). Voir notamment: Jean-Yves Fontaine, « Syndicalisme électronique en gendarmerie », Libération, 18 avril 2008.

\section{RÉSUMÉS}

L'article interroge la reconfiguration des rhétoriques de métiers, sous l'effet de l'appropriation des dispositifs d'auto-publication par les groupes professionnels. Pour cela, il étudie un corpus de cinq «blogs de policiers » édités sous pseudonyme par des policiers en exercice, par analyse de discours et entretiens. Leurs auteurs revendiquent un témoignage personnel et authentique sur le rôle et le travail des "policiers de base ». Pour soutenir cette promesse, ils façonnent et articulent différents ethè discursifs dans et par leurs écrits d'écran : le témoin, le confident, le spécialiste, l'auteur. Chacun correspond à une stratégie énonciative pour établir un lien avec les lecteurs, mais aussi pour se hisser au rang d'énonciateur légitime et parler de et au nom de la profession.

The paper deals with the reconfiguration of professional discourses, as a result of the use of blogs and social media by professional groups. Using both discourse analysis and research interviews, it studies a corpus of five blogs written by French policemen. Hidden behind pseudonyms, the authors tell their occupation and themselves candidly. They produce a first-person account of the condition of "street cops." For that purpose, they use discursive self-presentation strategies, building different images of themselves. These enunciation strategies aim to create a link with the readers, but also to appear as legitimate enunciators able to speak on behalf of the profession. 
INDEX

Mots-clés : analyse du discours, police, blog, témoignage, discours professionnel, ethos discursif Keywords : police, blog, first-person account, professional discourse, discourse analysis, selfpresentation

\section{AUTEURS}

\section{PIERRE-YVES CONNAN}

Cérep, Université de Reims Champagne-Ardenne

\section{GUILLAUME LE SAULNIER}

Cérep, Université de Reims Champagne-Ardenne

\section{BENOÎT VERDIER}

Cérep, Université de Reims Champagne-Ardenne 